\title{
Tumor targeting and imaging with dual- peptide conjugated multifunctional liposomal nanoparticles
}

This article was published in the following Dove Press journal:

International Journal of Nanomedicine

4 December 2013

Number of times this article has been viewed

\author{
Christine Rangger ${ }^{\prime}$ \\ Anna Helbok' \\ Jane Sosabowski \\ Christian Kremser ${ }^{3}$ \\ Gottfried Koehler ${ }^{4}$ \\ Ruth Prassl ${ }^{5,6}$ \\ Fritz Andreae ${ }^{7}$ \\ Irene JVirgolini' \\ Elisabeth von Guggenberg' \\ Clemens Decristoforo' \\ 'Department of Nuclear Medicine, \\ Innsbruck Medical University, \\ Innsbruck, Austria; ${ }^{2}$ Centre for \\ Molecular Oncology, Barts Cancer \\ Institute, Queen Mary University of \\ London, London, UK; ${ }^{3}$ Department \\ of Radiology, Innsbruck Medical \\ University, Innsbruck, ${ }^{4}$ Department \\ of Computational and Structural \\ Biology, Max Perutz Laboratories, \\ University of Vienna, Wien, ${ }^{5}$ Institute \\ of Biophysics, Medical University \\ of Graz, Graz, 'udwig Boltzmann \\ Institute for Lung Vascular Research, \\ ${ }^{7}$ piCHEM Research and Development, \\ Graz, Austria
}

Background: The significant progress in nanotechnology provides a wide spectrum of nanosized material for various applications, including tumor targeting and molecular imaging. The aim of this study was to evaluate multifunctional liposomal nanoparticles for targeting approaches and detection of tumors using different imaging modalities. The concept of dual-targeting was tested in vitro and in vivo using liposomes derivatized with an arginine-glycine-aspartic acid (RGD) peptide binding to $\alpha_{v} \beta_{3}$ integrin receptors and a substance $\mathrm{P}$ peptide binding to neurokinin-1 receptors.

Methods: For liposome preparation, lipids, polyethylene glycol building blocks, DTPAderivatized lipids for radiolabeling, lipid-based RGD and substance P building blocks and imaging labels were combined in defined molar ratios. Liposomes were characterized by photon correlation spectroscopy and zeta potential measurements, and in vitro binding properties were tested using fluorescence microscopy. Standardized protocols for radiolabeling were developed to perform biodistribution and micro-single photon emission computed tomography/computed tomography (SPECT/CT) studies in nude mice bearing glioblastoma and/or melanoma tumor xenografts. Additionally, an initial magnetic resonance imaging study was performed.

Results: Liposomes were radiolabeled with high radiochemical yields. Fluorescence microscopy showed specific cellular interactions with RGD-liposomes and substance P-liposomes. Biodistribution and micro-SPECT/CT imaging of ${ }^{111}$ In-labeled liposomal nanoparticles revealed low tumor uptake, but in a preliminary magnetic resonance imaging study with a single-targeted RGD-liposome, uptake in the tumor xenografts could be visualized.

Conclusion: The present study shows the potential of liposomes as multifunctional targeted vehicles for imaging of tumors combining radioactive, fluorescent, and magnetic resonance signaling. Specific in vitro tumor targeting by fluorescence microscopy and radioactivity was achieved. However, biodistribution studies in an animal tumor model revealed only moderate tumor uptake and no additive effect using a dual-targeting approach.

Keywords: liposomal nanoparticles, radiolabeling, dual-targeting, tumor imaging, multifunctionality

\section{Introduction}

Noninvasive imaging modalities, such as single photon emission computed tomography (SPECT), positron emission tomography, magnetic resonance imaging (MRI), optical fluorescence, or targeted ultrasound, are important tools in clinical diagnosis. They are widely used for monitoring of disease status and the real-time evaluation of treatment response. ${ }^{1-3}$

The design of targeting and imaging agents that allow early detection of cellular abnormalities is crucial to make pathologic changes visible, quantifiable, and
Correspondence: Clemens Decristoforo Department of Nuclear Medicine, Innsbruck Medical University,

Anichstrasse 35,

A-6020 Innsbruck, Austria

Tel +435125048095 I

Fax +435I2504678095I

Email clemens.decristoforo@uki.at 
traceable over time. For the efficient delivery of both targeting and imaging labels, a variety of carrier systems has been investigated. ${ }^{4}$ Due to their composition, liposomal nanoparticles (LNPs) form an excellent platform for the combination of imaging, diagnosis, and treatment of cancer. These spherical vesicles are composed of a bilayer of phospholipids with an aqueous interior and are able to accommodate lipophilic compounds in the lipidic bilayer and hydrophilic compounds in the aqueous compartment. ${ }^{1,5}$ Coated with polymers (eg, polyethylene glycol [PEG]) to improve in vivo stability and taking advantage of the enhanced permeation and retention effect, liposomes have shown great potential in the field of nanosized drug delivery systems. ${ }^{6-9}$

Combination with appropriate targeting moieties, such as peptides or antibodies, may allow specific concentration of nanoparticles in pathologic areas. Selection of targeting moieties specifically binding to diseased tissue while not affecting normal cells is essential for site-specific targeting. ${ }^{10}$ A very promising target in this respect is tumor-induced angiogenesis. Newly formed blood vessels show high expression of diverse specific molecules which are absent in normal vasculature. ${ }^{11}$ An example of such in vivo targets are $\alpha_{v} \beta_{3}$ integrin receptors, which are strongly overexpressed on the activated endothelium of angiogenic blood vessels. ${ }^{12}$ The $\alpha_{\mathrm{v}} \beta_{3}$ integrins are attached to extracellular matrix proteins exposing the tripeptide sequence arginine-glycine-aspartic acid (RGD) as a receptor recognition signal. ${ }^{13}$ We have recently developed radiolabeled LNPs carrying a cyclic RGD peptide showing more favorable binding characteristics than linear or multimeric RGD peptides. ${ }^{14,15}$

Another interesting target is the $G$ protein-coupled neurokinin-1 receptor interacting with the neuropeptide substance $\mathrm{P}$ (SP). This receptor, besides being overexpressed in several malignancies, including breast, ovarian, and prostate cancer as well as glioblastoma and melanoma, is also present on tumor cells infiltrating the intratumoral and peritumoral vasculature. ${ }^{16-18}$ The SP/neurokinin-1 receptor system plays an important role in the mitogenesis, cell migration, angiogenesis, and metastasis of the above-mentioned tumors. ${ }^{19}$ Therefore, it was decided to translate the knowledge obtained with RGD carrying LNPs to this alternative targeting system.

The availability of a tumor xenograft mouse model with human glioblastoma cells (U-87 MG), a cell line known to express both $\alpha_{\mathrm{v}} \beta_{3}$ integrins and $\mathrm{SP} /$ neurokinin-1 receptors, allowed us to investigate both targeting systems in the same animal model..$^{20,21}$ Additionally, tumor xenografts with human melanoma cells (M21) only expressing $\alpha_{\mathrm{v}} \beta_{3}$ integrin receptors were used. The current study describes for the first time the evaluation of multifunctional LNPs carrying both the RGD and SP peptide sequence. Such dual-targeting approaches could potentially lead to improvements in targeted delivery of drugs and may solve the frequent problem of low receptor density in vivo resulting in low accumulation at the targeted site. ${ }^{22}$

The targeted liposomes were also derivatized with labels for imaging purposes, thereby resulting in multifunctionalized nanoconstructs. Attaching a chelating group provided the basis for radiolabeling and subsequent microSPECT/CT studies in tumor-bearing nude mice but also allowed gadolinium-loading for a preliminary MRI study. Derivatization with a fluorescent label (Rhodamine-B) made it possible to perform the characterization at the cellular level by means of fluorescence microscopy.

\section{Materials and methods}

All reagents were purchased from VWR International (Radnor, PA, USA) or Sigma-Aldrich (St Louis, MO, USA) and were of analytical grade. Unless stated otherwise, reagents were used as supplied with no further purifications.

1,2-dimyristoyl-sn-glycero-3-phosphoethanolamine$\mathrm{N}$-diethylenetriaminepentaacetic acid (DMPE-DTPA), 1-palmitoyl-2-oleoyl-sn-glycero-3-phosphocholine (POPC), 1,2-dipalmitoyl-sn-glycero-3-phosphoethanolamine-N(lissamine Rhodamine-B sulfonyl), 1,2-dimyristoyl-sn-glycero3-phosphoethanolamine-N-diethylenetriaminepentaacetic acid (gadolinium salt), and the Avanti ${ }^{\circledR}$ Mini-Extruder for liposome preparation were obtained from Avanti Polar Lipids Inc. (Alabaster, AL, USA). Cholesterol was purchased from Sigma-Aldrich. ${ }^{111}$ In chloride was obtained either from Mallinckrodt Medical BV (Petten, the Netherlands) or PerkinElmer (Waltham, MA, USA). Radioactivity measurements of the different samples were carried out using the 2480 Wizard $^{2}$ gamma counter from PerkinElmer.

Cell culture media (Gibco ${ }^{\circledR}$ Roswell Park Memorial Institute 1640 [RPMI 1640], Gibco Dulbecco's Modified Eagle Medium [DMEM]; Life Technologies, Carlsbad, CA, USA), trypsin solution $(2.5 \%, 10 \times$, liquid), sodium pyruvate solution (100 mM, liquid), Gibco fetal bovine serum (Life Technologies), minimum essential medium non-essential amino acid solution (100×, liquid), and penicillin/streptomycin/ glutamine solution $(100 \times$, liquid) were sourced from Thermo Fisher Scientific (Waltham, MA, USA). Human glioblastoma cells (U-87 MG) were purchased from the European Collection of Cell Cultures (Salisbury, UK). Human melanoma cells 
(M21) were a kind gift from Dr David A Cheresh and the Scripps Research Institute (La Jolla, CA, USA).

\section{Synthesis of targeted building blocks Preparation of DSPE-PEG(2000)-Mal-RGD}

Synthesis of the S-acetyl-3-mercaptopropionic acid (SAMA)RGD building block and crosslinking with 1,2-distearoyl-snglycero-3-phosphoethanolamine-N-(maleimide[PEG]2000) (DSPE-PEG[2000]-Mal) was performed as described previously. ${ }^{14}$ Briefly, SAMA-RGD was synthesized using the solid phase peptide synthesis technique with preloaded Fmoc-Gly-2-Cl-trityl resin and hydroxybenzotriazole/1,3diisopropylcarbodiimide (HOBt/DIC) in situ activation. Using a mixture of $20 \%$ 1,1,1,3,3,3-hexafluoro-2-propanol in dichloromethane, the linear side chain-protected peptide was cleaved from the resin. The crude product, $\mathrm{H}-\mathrm{Asp}(\mathrm{OtBu})-$ dTyr(tBu)-Lys(Dde)-Arg(Pbf)-Gly-OH, was then purified by high-performance liquid chromatography (HPLC) and freeze dried. The $C-N$-cyclization of the linear protected peptide was done in acetonitrile using benzotriazol-1-yloxy-tris-pyrrolidino-phosphonium hexafluorophosphate. The Dde-protection group of lysine was removed with $2 \%$ hydrazine hydrate in 1-methyl-2-pyrrolidinone and the crosslinker SAMA was attached onto the side chain amino group of lysine using preactivated SAMA-pentafluorophenylester. Each cleavage, cyclization, or modification step was finalized by reversed-phase HPLC purification and lyophilization. SAMA-RGD and DSPE-PEG(2000)-Mal was crosslinked by in situ deprotection of the S-acetyl group with hydroxylamine $\left(\mathrm{NH}_{2} \mathrm{OH}\right)$, releasing a free thiol moiety which then reacts with the maleimide group of the DSPE-PEG(2000)-Mal. SAMA-RGD and DSPE-PEG(2000)-Mal were dissolved in methanol at a molar peptide-to-lipid ratio of 1.5:1. After addition of a 200-fold molar excess of $\mathrm{NH}_{2} \mathrm{OH}$ over SAMA-RGD (dissolved in 0.1 M phosphate-buffered saline [pH 6.0]), the mixture was left to react for 4 hours at room temperature and was finally freeze dried. Completeness of product formation was monitored by reversed-phase HPLC and mass spectrometry. Using this approach, the lipid was completely converted and no further work step was needed, since unreacted peptide was removed during liposome formation.

\section{Preparation of DSPE-PEG(2000)-Mal-SP}

Similar to the SAMA-RGD building block, the SP building block was synthesized using the solid phase peptide synthesis technique with a commercially available batch synthesizer, a TentaGel ${ }^{\circledR} \mathrm{S}$ RAM resin, and HOBt/DIC in situ activation. The $N$-terminal residue of cysteine was linked to the remaining peptide via a (2-[2-(Fmoc-amino)ethoxy] ethoxy) acetic acid spacer. When the peptide elongation was completed, the crude product was cleaved from the resin using a $92 \%$ trifluoroacetic acid $/ 8 \%$ scavenger mixture (ethylenediaminetetraacetic acid, thioanisole, water, triisopropylsilane). The product was then purified by preparative reversed-phase HPLC, and pure fractions were freeze dried. For crosslinking of Cys-O2O-SP and DSPE-PEG(2000)-Mal, both compounds were dissolved in a mixture of $650 \mu \mathrm{L}$ methanol, $750 \mu \mathrm{L}$ of conjugation buffer (0.1 M o-phosphoric acid, 0.9 M sodium chloride, $17 \mathrm{mM}$ ethylenediaminetetraacetic acid; $\mathrm{pH} 7.2), 1,050 \mu \mathrm{L}$ of water, $200 \mu \mathrm{L}$ of dimethyl sulfoxide, and $100 \mu \mathrm{L}$ of chloroform at a peptide-to-lipid molar ratio of 1.5:1 $\left(3.1 \times 10^{-6}\right.$ mol Cys-O2O-SP:2.1 × 10 ${ }^{-6}$ mol DSPE-PEG[2000]-Mal). Subsequently, the mixture was left to react for 4 hours at room temperature. Product formation was monitored by reversed-phase HPLC and mass spectrometry. Residual amounts of unreacted SP were removed by dissolving the building block in chloroform and the product was again monitored by reversed-phase HPLC, confirming a purity of more than $95 \%$.

\section{Liposome preparation and characterization}

Liposomes were prepared using the lipid film hydration method described by Bangham et al followed by extrusion. ${ }^{23}$ Stock solutions (resulting in a final lipid content of $30 \mathrm{mg} / \mathrm{mL}$ ) of POPC, cholesterol, DSPE-PEG(2000), and, for radiolabeling, a phospholipid coupled to the chelating agent (DMPE-DTPA) were prepared and dissolved in pure chloroform. Depending on the liposome type, different targeting sequences (RGD and/or SP) and imaging labels for fluorescence microscopy and MRI were used. Volumes of the above-mentioned components were mixed in predetermined molar ratios in a small round bottom glass vial. Subsequently, the organic solvent was evaporated under a stream of nitrogen, achieving a dry lipid film that was completely dried in vacuo overnight. Hydration of the film was obtained by adding $1 \mathrm{~mL}$ of $6.6 \mathrm{mM}$ disodium phosphate dihydrate/1.4 mM monopotassium phosphate/136.9 mM sodium chloride phosphate-buffered saline $(\mathrm{pH} 7.4)$ and repeated vortexing for 60 minutes at $40^{\circ} \mathrm{C}$. Afterwards, the lipid suspension was extruded 21 times through a polycarbonate filter (100 nm pore size) using the Avanti Mini-Extruder. Based on extensive in vitro and in vivo stability studies by our group, the liposomal suspensions were always stored at $4^{\circ} \mathrm{C}$ for no longer than one month to guarantee stable liposomes without aggregates. ${ }^{24}$ 
Abbreviations used for the various LNPs are as follows: RLPs (liposomes carrying the RGD building block), SP-LPs (liposomes derivatized with SP), Hybrid-LPs (liposomes containing both targeting sequences [dual-targeted]), and NoTarget-LPs (liposomes without targeting properties). Liposomes carrying the fluorescent label Rhodamine-B were abbreviated as "RhB-LNPs" and when derivatized with gadolinium for MRI they were labeled as "Gd-LNPs". The composition of the liposomal formulations in terms of molar ratios of the building blocks and targeting sequences is presented in Table 1.

The size and polydispersity index of the liposomes were determined by photon correlation spectroscopy using a submicron particle sizer Nicomp ${ }^{\text {TM }} 380$ (Particle Sizing Systems, Port Richey, FL, USA) equipped with a $15 \mathrm{~mW}$ laser diode and a photomultiplier tube detector with an optical fiber set at 90 degrees for detection of the scattered light from the probe. Additionally, zeta potential measurements were performed using the Nicomp 380 ZLS particle sizer at $23^{\circ} \mathrm{C}$ and an E-field strength of $5 \mathrm{mV}$. For data acquisition, PSS ZPW388 version 1.65 software (Particle Sizing Systems) was used.

\section{Radiolabeling of liposomes}

For radiolabeling, ${ }^{111} \mathrm{In}$ chloride $(5-100 \mathrm{MBq} / \mathrm{mL})$ was diluted in $0.05 \mathrm{M}$ hydrogen chloride to a volume of $50 \mu \mathrm{L}$. Subsequently, $50 \mu \mathrm{L}$ of gentisic acid $(0.24 \mathrm{M}) /$ sodium acetate buffer (0.4 M; pH 4.5) and $100 \mu \mathrm{L}$ of the liposomal suspension were added, obtaining a total reaction volume of $200 \mu \mathrm{L}$. The labeling solution was then allowed to react for 30 minutes at room temperature.

Radiolabeling efficiency (radiochemical yield) was identified by instant thin layer chromatography on silica gel strips (Agilent Technologies, Santa Clara, CA, USA). Samples were developed using two different mobile phases, ie, $1 \mathrm{M}$ ammonium acetate/ $0.05 \mathrm{M}$ ethylenediaminetetraacetic acid and acetic acid/pyridine/water (5/3/1.5). Using the $1 \mathrm{M}$ ammonium acetate/0.05 $\mathrm{M}$ ethylenediaminetet- raacetic acid system, labeled product and radiocolloid stay at the origin (retention factor radiolabeled LNP and radiocolloid $=0.0-0.3$ ), whereas the "free" radionuclide migrates with the solvent front (retention factor "free" radionuclide $=0.8-1.0$ ). Using the acetic acid/pyridine/ water (5/3/1.5) system, labeled product and "free" radionuclide migrate with the solvent front (retention factor radiolabeled LNP and "free" radionuclide $=0.8-1.0$ ) and the radiocolloid remains at the origin (retention factor radiocolloid $=0.0-0.2$ ). The distribution of radioactivity was analyzed using a Cyclone ${ }^{\circledR}$ Plus Phosphor Imager (PerkinElmer).

\section{Cell culture}

U-87 MG cells were cultured in tissue culture flasks (Cellstar $^{\circledR}$; Greiner Bio-One, Kremsmuenster, Austria) using DMEM supplemented with 10\% volume/volume $(\mathrm{v} / \mathrm{v})$ heat-inactivated fetal bovine serum, $1 \% \mathrm{v} / \mathrm{v}$ penicillin/ streptomycin/glutamine solution, $1 \% \mathrm{v} / \mathrm{v}$ sodium pyruvate solution, and $1 \% \mathrm{v} / \mathrm{v}$ nonessential amino acid solution. M21 cells were also grown in tissue culture flasks using DMEM supplemented with $10 \% \mathrm{v} / \mathrm{v}$ heat-inactivated fetal bovine serum and $1 \% \mathrm{v} / \mathrm{v}$ penicillin/streptomycin/glutamine solution. Both cell lines were grown to confluence at $37^{\circ} \mathrm{C}$ in a humidified atmosphere of $95 \%$ air $/ 5 \%$ carbon dioxide and split every 48 hours.

For the fluorescence microscopy studies, mouse fibroblast cells (L-929) were cultured in Minimal Essential Medium supplemented with $10 \% \mathrm{v} / \mathrm{v}$ heat-inactivated fetal bovine serum, $1 \% \mathrm{v} / \mathrm{v}$ L-glutamine, $1 \% \mathrm{v} / \mathrm{v}$ nonessential amino acids, and $1 \% \mathrm{v} / \mathrm{v}$ antibiotic/antimycotic in tissue culture flasks. The cells were grown to confluence at $37^{\circ} \mathrm{C}$ in a humidified atmosphere of $95 \%$ air $/ 5 \%$ carbon dioxide.

\section{Fluorescence microscopy}

For laser scanning imaging, a Zeiss LSM 510 (Carl Zeiss Meditec AG,) microscope was used. Two cancer cell lines (U-87 MG, M21) and a negative control mouse fibroblast

Table I Composition of different liposomal formulations: molar ratios of the building blocks and targeting sequences

\begin{tabular}{lllllll}
\hline Liposome type & Cholesterol & POPC & DMPE-DTPA & DSPE-PEG(2000) & RGD & Substance P \\
\hline RLP & 2 & 3 & 0.3 & 0.3 & $0.3(6 \mathrm{~mol} \%)$ & \\
SP-LP & 2 & 3 & 0.3 & 0.3 & $0.3(6 \mathrm{~mol} \%)$ \\
Hybrid-LP & 2 & 3 & 0.3 & 0.3 & $0.015(0.3 \mathrm{~mol} \%)$ & $0.015(0.3 \mathrm{~mol} \%)$ \\
NoTarget-LP & 2 & 3 & 0.3 & 0.3 & - & - \\
\hline
\end{tabular}

Abbreviations: DMPE, I,2-dimyristoyl-sn-glycero-3-phosphoethanolamine; DSPE, I,2-distearoyl-sn-glycero-3-phosphoethanolamine; DTPA, diethylenetriaminepentaacetic acid; Hybrid-LP, liposomal nanoparticle carrying an RGD and SP building block; LP, liposome; NoTarget-LP, liposomal nanoparticle with no targeting sequence; PEG, polyethylene glycol; POPC, I-palmitoyl-sn-glycero-3-phosphocholine; RGD, arginine-glycine-aspartic acid; RLP, liposomal nanoparticle carrying an RGD building block; SP, substance P; SP-LP, liposomal nanoparticle carrying an SP building block. 
cell line (L-929) were grown overnight in LabTek ${ }^{\mathrm{TM}}$ chamber slides (Nunc, Langensebold, Germany) until a growth density of about $40-50 \%$ was reached. The Rhodamine-B-derivatized liposomes (RhB-RLP, RhB-SP-LP, RhB-Hybrid-LP, RhBNoTarget-LP) were diluted 1:250 times with the appropriate cell culture medium and incubated together with the cells for 15 minutes. After incubation, the liposome suspension was removed and the cells were washed twice with phosphatebuffered saline. Cells were then stained with Onkovidon ${ }^{\circledR}$ green life cell lipid staining kit solution (Onko Tek GmbH, Vienna, Austria) for 10 minutes to make the plasma membrane visible. After another washing step with phosphatebuffered saline, the cells were imaged for 15-20 minutes. When so-called blebbing occurred (formation of irregular bulges in the plasma membrane due to prolonged irradiation with laser beam) the imaging was stopped immediately. For all cases, full stacks of images could be obtained.

\section{In vivo experiments}

\section{Biodistribution studies}

All animal experiments were in compliance with the Austrian animal protection laws and conducted with the approval of the Austrian Ministry of Science (BMWF-66.011/0147II/10b/2008). For the animal studies, 6-week-old, female athymic BALB/c nude mice (Charles River Laboratories International, Inc., Sulzfeld, Germany) were used. The mice were kept under pathogen-free conditions on a normal ad libitum diet. For induction of tumor xenografts, $2.5 \times 10^{6}$ U-87 MG cells were injected subcutaneously into the left hind limb. Additionally, for studies with Hybrid-LPs, $5 \times 10^{6}$ M21 cells were injected in the right hind limb of the mouse (U-87 MG tumor xenograft on the left hind limb and M21 tumor xenograft on the right hind limb of the same mouse). Tumor size and the state of health of the animals were checked regularly. Approximately 3 weeks after inoculation, tumors had reached a volume of about $0.2-0.5 \mathrm{~mL}$ and appropriate animals were chosen for the experiments.

On the day of the experiment, a total volume of $150 \mu \mathrm{L}$ (15 MBq ${ }^{111} \mathrm{In} / \mathrm{kg}$ bodyweight; $12 \mu \mathrm{g}$ lipid/mouse) of ${ }^{111} \mathrm{In}$ labeled LNP (RLP, SP-LP, Hybrid-LP) was injected intravenously into a lateral tail vein of the mouse (RLP, $n=7$; SP-LP, $\mathrm{n}=7$; Hybrid-LP, $\mathrm{n}=10$; minimum $\mathrm{n}=3$ per time point). The mice were kept warm during the incubation time to avoid hypothermia. At 1 hour or 4 hours post-injection, the mice were sacrificed by cervical dislocation without anesthesia by a trained person using appropriate equipment. Tissues (blood, muscle), organs (heart, lung, liver, spleen, pancreas, stomach, intestine, kidneys), and tumors were collected into plastic vials and weighed. Stomach contents were removed and the organs then weighed. Standards were prepared from a sample of the injected radioligand. The accumulated activity in the various probes was measured using a gamma counter and the percentage of injected dose per gram organ/tissue $(\% \mathrm{ID} / \mathrm{g})$ was calculated for each sample.

\section{Micro-SPECT/CT imaging studies}

Initial imaging studies were performed at the Centre for Molecular Oncology, Barts Cancer Institute, Queen Mary University of London (London, UK). Tumor xenografts were induced in three female beige severe combined immunodeficiency mice by subcutaneous injection of $2.5 \times 10^{6} \mathrm{U}-87 \mathrm{MG}$ cells into the left hind limb of the mouse. The animals were used when tumors had reached a volume of about $0.2-0.5 \mathrm{~mL}$. Subsequently, each mouse was injected with $12 \mathrm{MBq}$ of

${ }^{111}$ In-labeled LNP (RLP, Hybrid-LP, NoTarget-LP) via a lateral tail vein. For imaging, the mice were anesthetized (4\% isoflurane and $0.5-1 \mathrm{~L}$ per minute oxygen) and imaged using a NanoSPECT/CT in vivo preclinical imager (Bioscan Inc., Washington, DC, USA) fitted with $2 \mathrm{~mm}$ pinhole collimators in helical scanning mode (20 projections, 30 minutes). Corresponding CT images were acquired with a $45 \mathrm{kVP}$ $\mathrm{X}$-ray source in 180 projections over 10 minutes. All images were reconstructed in a $256 \times 256$ matrix using proprietary InVivoScope ${ }^{\mathrm{TM}}$ (Bioscan) software and fused using PMOD ${ }^{\mathrm{TM}}$ (Mediso Medical Imaging Systems, Budapest, Hungary).

\section{MRI studies}

About 2 weeks before the MRI studies, two BALB/c nude mice were injected subcutaneously with $5 \times 10^{6} \mathrm{M} 21$ cells into the right hind limb and $2.5 \times 10^{6} \mathrm{U}-87 \mathrm{MG}$ cells into the left hind limb of the same mouse. When tumors had reached a volume of about $0.2-0.5 \mathrm{~mL}$, appropriate animals were chosen for the experiments. For anesthesia, a freshly prepared mixture of $1 \mathrm{~mL}$ ketamine $(100 \mathrm{mg} / \mathrm{mL}), 0.5 \mathrm{~mL}$ xylazine $(20 \mathrm{mg} / \mathrm{mL})$, and $8.5 \mathrm{~mL}$ sodium chloride solution was used. After a single intraperitoneal injection of $200 \mu \mathrm{L}$ ketamine/ xylazine/sodium chloride mixture $(100 \mu \mathrm{L}$ per $10 \mathrm{~g}$ of body weight), the mice remained under anesthesia/sedation for about 40-50 minutes. If required, anesthesia was prolonged by injection of an additional amount of ketamine/xylazine/ sodium chloride mixture. To prevent drying of the corneas, an ophthalmic ointment was applied to the eyes of the mouse. All mice were kept warm to avoid hypothermia.

MRI of Gd-RLP and Gd-NoTarget-LP was performed on a clinical 3-T system equipped with a $40 \mathrm{mT} / \mathrm{m}$ (slew rate $200 \mathrm{~T} / \mathrm{m} / \mathrm{s}$ ) gradient system (Magnetom Verio, 
Siemens Health Care, Erlangen, Germany). Anesthetized mice were placed prone inside two small loop coils (standard finger coils) with an inner diameter of $30 \mathrm{~mm}$. After fast localizing scans, imaging was performed using a volume-interpolated three-dimensional T1 mapping sequence based on the variable flip angle method with the following parameters: repetition time $6.98 \mathrm{msec}$; spin echo time $2.48 \mathrm{msec}$; flip angle $1,3^{\circ}$; flip angle $2,15^{\circ}$; field of view $70 \times 140 \mathrm{~mm}$; acquisition matrix $92 \times 256$, interpolated by $\mathrm{k}$-space zero filling to $184 \times 512$; voxel size $0.27 \times 0.27 \times 1.8 \mathrm{~mm}$; number of slices 20 ; and number of averages $8 . .^{25,26}$ The total facility time required per mouse was approximately 10 minutes. Images were conducted before injection of Gd-LNPs and at one hour and 2.5/3 hours post-injection, and were analyzed off-line using ImageJ (Wayne Rasband, National Institutes of Health, Bethesda, MD, USA) whereby R1 parameter maps ( 1 1 =1/T1) were calculated from the acquired variable flip-angle images. Tumor and liver tissue were manually segmented on the R1 maps and R1 values for all voxels within the different tissues were recorded for the two time points. Enhancement of Gd-LNPs is expected to lead to an increase of R1 values in the respective tissue proportional to the nanoparticle concentration $\left(\Delta \mathrm{R} 1=\mathrm{r}^{*} \mathrm{c} ; \mathrm{r}\right.$, relaxivity of the nanoparticles; c, concentration of Gd-LNPs).

\section{Statistical analysis}

All data were expressed as the mean \pm standard deviation. The data from the biodistribution studies in tumor-xenografted $\mathrm{BALB} / \mathrm{c}$ nude mice were compared using an unpaired $t$-test with a significance level of $P<0.05$. All analyses were performed using Microsoft Office XP Professional Excel ${ }^{\circledR}$ version 10.0.6626.0 (Microsoft Corporation, Redmond, WA, USA). In the MRI studies, the Shapiro-Wilkinson normality test was used to show that the data were not normally distributed. The significance of the R1 increase between the two time points was evaluated using an unpaired, one-sided Wilcoxon rank sum test with the $\mathrm{R}$ Project for Statistical Computing ( $\mathrm{R}$ version 2.15.0).

\section{Results}

\section{Liposome characterization} and radiolabeling

The particle size distribution for all tested LNPs was highly homogenous and results were reproducible with only minor deviations. Mean size and polydispersity index values were as follows: $110.4 \pm 4.3 \mathrm{~nm}$ for RLPs (polydispersity index $0.035 ; \mathrm{n}=3$ ), $125.4 \pm 1.8 \mathrm{~nm}$ for SP-LPs (polydispersity index $0.014 ; \mathrm{n}=2$ ), $131.3 \pm 5.3 \mathrm{~nm}$ for Hybrid-LPs (polydispersity index $0.124 ; \mathrm{n}=2$ ), and $117.7 \pm 5.0 \mathrm{~nm}$ for NoTarget-LPs (polydispersity index $0.011 ; n=2)$. Zeta potential measurements revealed values between $-2 \mathrm{mV}$ and $3 \mathrm{mV}(\mathrm{n}=9)$.

It was possible to label all LNPs with ${ }^{111}$ In chloride. The following radiochemical yield values were obtained: $97.6 \% \pm 1.7 \%$ for RLPs $(n=6) ; 97.4 \% \pm 1.9 \%$ for SP-LPs $(\mathrm{n}=7) ; 97.7 \% \pm 1.7 \%$ for Hybrid-LPs $(\mathrm{n}=3)$; and $97.2 \% \pm 1.2 \%$ for NoTarget-LPs $(n=4)$. Lipid composition and particle size did not influence radiochemical yield values, but for liposome solutions older than 3 months, radiochemical yield values dropped significantly. For this reason, only freshly prepared LNPs (with less than one month storage) were used.

\section{Fluorescence microscopy}

Incubation of M21 and U-87 MG cells with RhB-NoTarget-LP resulted in no detectable liposome-to-cell association, and no binding was found for any RhB-LNP with the negative control cell line L-929. The most pronounced cell surface binding was observed with RhB-RLP on both M21 and U-87 MG cells and RhB-SP-LP on U-87 MG cells, which reflects the expression level of the receptors on these cells. For RhB-Hybrid-LP, only few interactions were found with the different tumor cell lines and no synergistic effect of dual-targeting was observed. Images of representative fluorescence microscopy samples are shown in Figure 1, indicating specific binding of the targeted LNPs to the cancer cell lines.

\section{In vivo experiments}

\section{Biodistribution studies}

${ }^{111}$ In-RLPs and ${ }^{111}$ In-SP-LPs showed comparable uptake in the organs and tissues of U-87 MG tumor-xenografted BALB/c nude mice. For ${ }^{111}$ In-SP-LP, major uptake was found in the liver, with values of $39.7 \% \pm 8.7 \% \mathrm{ID} / \mathrm{g}$. Liver uptake of ${ }^{111} \mathrm{In}-\mathrm{RLP}$ was higher with $46.0 \% \pm 4.9 \% \mathrm{ID} / \mathrm{g}$ ( 1 hour post-injection). At 4 hours post-injection, values increased to $57.1 \% \pm 1.2 \% \mathrm{ID} / \mathrm{g}$ for ${ }^{111} \mathrm{In}-\mathrm{SP}-\mathrm{LP}$ and to $51.4 \% \pm 10.1 \% \mathrm{ID} / \mathrm{g}$ for ${ }^{111} \mathrm{In}$-RLP. Blood levels at 1 hour post-injection were $7.9 \% \pm 1.1 \% \mathrm{ID} / \mathrm{g}$ for ${ }^{111} \mathrm{In}-\mathrm{SP}-\mathrm{LP}$ and $10.2 \% \pm 0.9 \% \mathrm{ID} / \mathrm{g}$ for ${ }^{111} \mathrm{In}-\mathrm{RLP}$, and decreased to $3.2 \% \pm 0.2 \% \mathrm{ID} / \mathrm{g}$ for ${ }^{111} \mathrm{In}-$ SP-LP and to $1.9 \% \pm 0.1 \% \mathrm{ID} / \mathrm{g}$ for ${ }^{111} \mathrm{In}-\mathrm{RLP}$ at 4 hours post-injection. Nonspecific uptake in the other tissues (heart, stomach, pancreas, intestine, kidneys) was $<5 \%$ ID/g at 1 hour post-injection for both ${ }^{111}$ In-labeled LNPs. Only uptake in the lung was slightly higher, with $6.7 \% \pm 1.2 \% \mathrm{ID} / \mathrm{g}$ for ${ }^{111}$ In-SP-LP and $8.9 \% \pm 0.4 \%$ ID/g for ${ }^{111} \mathrm{In}$-RLP (Figure 2). Receptor specific uptake in the U-87 MG tumor xenografts was moderate for both ${ }^{111} \mathrm{In}-\mathrm{SP}-\mathrm{LP}(1.0 \% \pm 0.3 \% \mathrm{ID} / \mathrm{g})$ and 

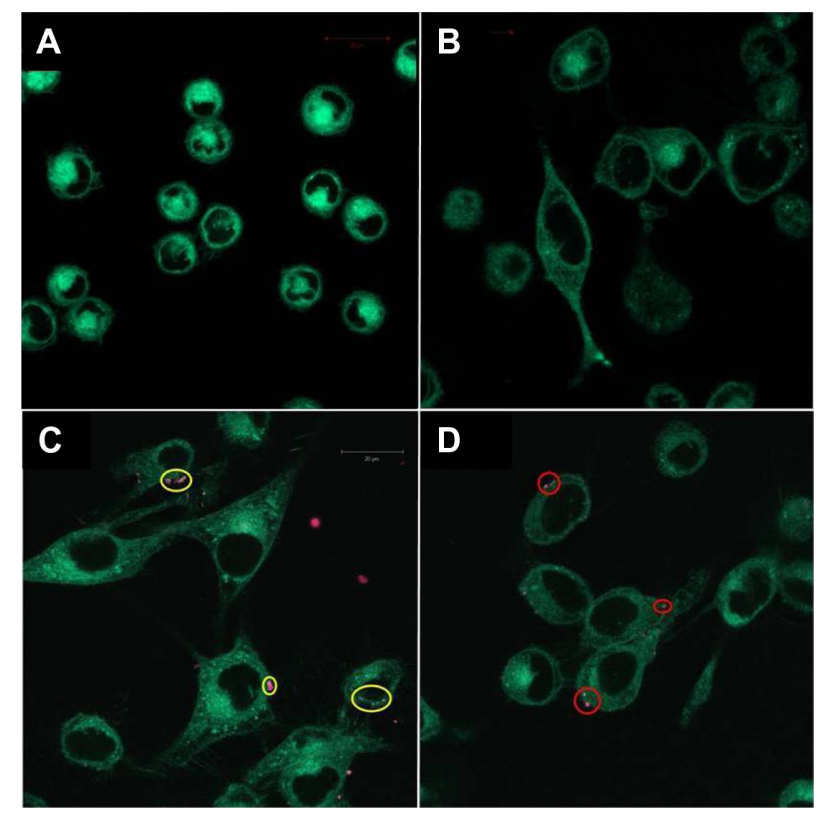

Figure I Fluorescence microscopy images of different cell lines incubated with RhB-labeled liposomal nanoparticles. (A) Negative control cell line L-929 showed no binding of RhB-Hybrid-LPs. (B) M2I cells incubated with RhB-NoTarget-LP indicated no cellular interactions. (C) Image of $M 2 I$ cells showing binding of RhBRLP (yellow circles). (D) Image of U-87 MG cells showing cellular interactions with RhB-SP-LP (red circles).

Abbreviations: Hybrid-LP, liposomal nanoparticle carrying an RGD and SP building block; LP, liposome; NoTarget-LP, liposomal nanoparticle with no targeting sequence; RGD, arginine-glycine-aspartic acid; RhB, Rhodamine-B; RLP, liposomal nanoparticle carrying an RGD building block; SP, substance P.
${ }^{111}$ In-RLP $(1.6 \% \pm 0.3 \% \mathrm{ID} / \mathrm{g})$ at 1 hour post-injection. Tumor uptake values of both ${ }^{111}$ In-LNPs revealed no significant difference between the two time points (Table 2).

The ${ }^{111} \mathrm{In}$-Hybrid-LP ex vivo distribution values of BALB/c nude mice bearing both U-87 MG and M21 tumor xenografts were as follows: liver uptake increased from $40.1 \% \pm 7.2 \% \mathrm{ID} / \mathrm{g}$ ( 1 hour post-injection) to $54.4 \% \pm 12.4 \% \mathrm{ID} / \mathrm{g}$ ( 4 hours postinjection). Blood levels declined from $20.4 \% \pm 4.3 \% \mathrm{ID} / \mathrm{g}$ (1 hour post-injection) to $6.0 \% \pm 0.5 \% \mathrm{ID} / \mathrm{g}$ ( 4 hours postinjection) and lung uptake dropped from $14.8 \% \pm 3.1 \% \mathrm{ID} / \mathrm{g}$ ( 1 hour post-injection) to $7.2 \% \pm 0.8 \% \mathrm{ID} / \mathrm{g}$ ( 4 hours postinjection). Uptake in the heart, stomach, pancreas, intestine, and kidneys at 4 hours post-injection was $<7.5 \%$ ID $/ g$ (Figure 3). Receptor specific tumor uptake was only moderate, with $0.6 \% \pm 0.1 \% \mathrm{ID} / \mathrm{g}$ for the $\mathrm{M} 21$ tumor xenograft and $1.0 \% \pm 0.6 \% \mathrm{ID} / \mathrm{g}$ for the U-87 MG tumor xenograft (1 hour post-injection). However, a significant difference $(P<0.02)$ was observed in tumor uptake in favor of the U-87 MG tumor xenografts (Table 2).

\section{Micro-SPECT/CT imaging studies}

Static imaging was performed on U-87 MG tumor xenograft-bearing female beige severe combined immunodeficiency mice after intravenous injection of either ${ }^{111}$ In-RLP,

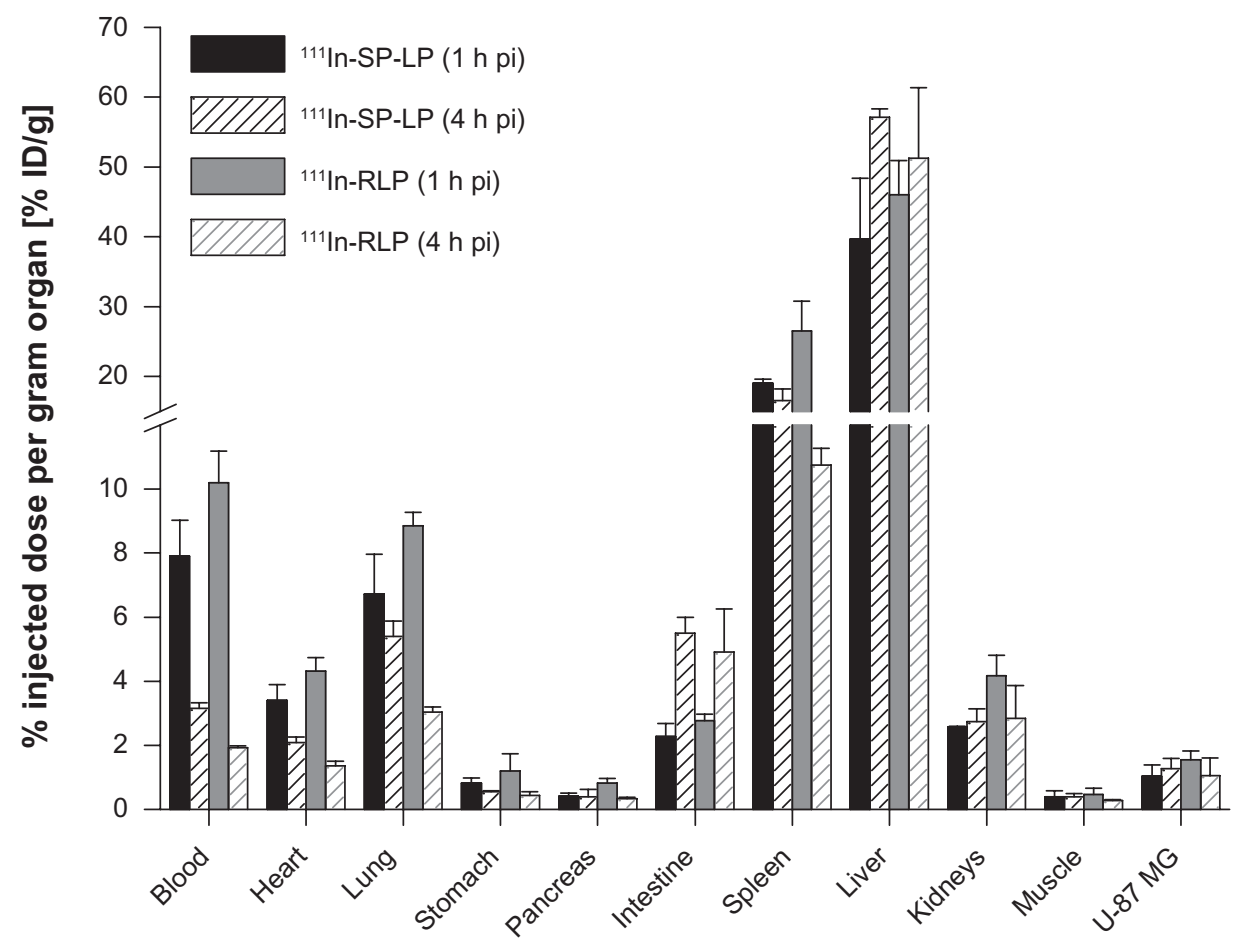

Figure 2 Ex vivo biodistribution of "'In-SP-LP ( $n=7)$ and "'In-RLP $(n=7)$ in BALB/c nude mice bearing U-87 MG tumor xenografts at I and 4 hours post-injection. Notes: Values are expressed as the percentage of injected dose per gram organ (\% ID/g; means \pm standard deviation).

Abbreviations: \% ID/g, percentage injected dose per gram organ; "'In, indium-III; h, hour; LP, liposome; pi, post-injection; RGD, arginine-glycine-aspartic acid; RLP, liposomal nanoparticle carrying an RGD building block; SP, substance P; SP-LP, liposomal nanoparticle carrying an SP building block. 
Table 2 Comparison of ex vivo tumor uptake of "'In-labeled RLP, SP-LP, and Hybrid-LP in BALB/c nude mice bearing U-87 MG and M2I tumor xenografts at I and 4 hours post-injection (RLP, n=7; SP-LP, n=7; Hybrid-LP, $n=10$ )

\begin{tabular}{|c|c|c|c|c|c|c|}
\hline & \multicolumn{3}{|c|}{$\%$ ID/g I hour post-injection } & \multicolumn{3}{|c|}{$\%$ ID/g 4 hours post-injection } \\
\hline & II'In-RLP & I'IIn-SP-LP & I'In-Hybrid-LP & I'In-RLP & II'In-SP-RLP & I'IIn-Hybrid-LP \\
\hline U-87 MG & $1.55 \pm 0.27$ & $1.04 \pm 0.34$ & $\mathrm{I} .03 \pm 0.6 \mathrm{I}$ & $1.05 \pm 0.56$ & $1.27 \pm 0.32$ & $1.23 \pm 0.28$ \\
\hline M2I & - & - & $0.55 \pm 0.10$ & - & - & $0.59 \pm 0.86$ \\
\hline
\end{tabular}

Note: Values are expressed as \% ID/g (means \pm standard deviation) at one and 4 hours post-injection.

Abbreviations: \% ID/g, percentage injected dose per gram of tissue; "'In, indium-III; Hybrid-LP, liposomal nanoparticle carrying an RGD and SP building block; LP, liposome; NoTarget-LP, liposomal nanoparticle with no targeting sequence; RGD, arginine-glycine-aspartic acid; RLP, liposomal nanoparticle carrying an RGD building block; SP, substance P; SP-LP, liposomal nanoparticle carrying an SP building block.

${ }^{111}$ In-Hybrid-LP, or ${ }^{111}$ In-NoTarget-LP at 4, 24, 48, and 72 hours. Overall, the results were comparable with values for the biodistribution studies. The main accumulation of activity for all ${ }^{111}$ In-labeled LNPs was found in the liver, spleen, and intestine, and nonspecific uptake in the heart, stomach, pancreas, and kidneys was low. Compared with the targeted LNPs ( ${ }^{111}$ In-RLP, ${ }^{111} \mathrm{In}$-Hybrid-LP), the ${ }^{111}$ In-NoTarget-LP showed higher accumulation of activity in the spleen and bladder (24 hours post-injection; Figure 4). Uptake of ${ }^{111}$ In-RLP and ${ }^{111}$ In-Hybrid-LP in the U-87 MG tumor xenografts was low and could not be clearly visualized at any time point.

\section{MRI studies}

For Gd-NoTarget-LP, no R1 increase was detected in either tumor xenografts at 3 hours post-injection (Figure 5). Instead, a significant increase $\left(P<2.2 \times 10^{-16}\right)$ of R1 values within the liver could be observed at 1 hour and 3 hours post-injection, respectively. These observations suggest that the Gd-NoTarget-LP did not accumulate within the tumor xenografts, but was instead taken up by the liver. For the targeted Gd-RLP, slightly different behavior was observed depending on the tumor model. The U-87 MG tumor xenograft showed a steady increase in R1 over time, whereas the M21 tumor xenograft showed a highly significant increase only at 2.5 hours postinjection, suggesting that the Gd-RLP accumulated within both tumor xenografts (Figure 6). A R1 increase for Gd-RLP could not be detected within the liver tissue in the 2.5-hour observation period.

\section{Discussion}

An important goal in clinical diagnostics is the noninvasive detection of biological markers in diseased tissue to

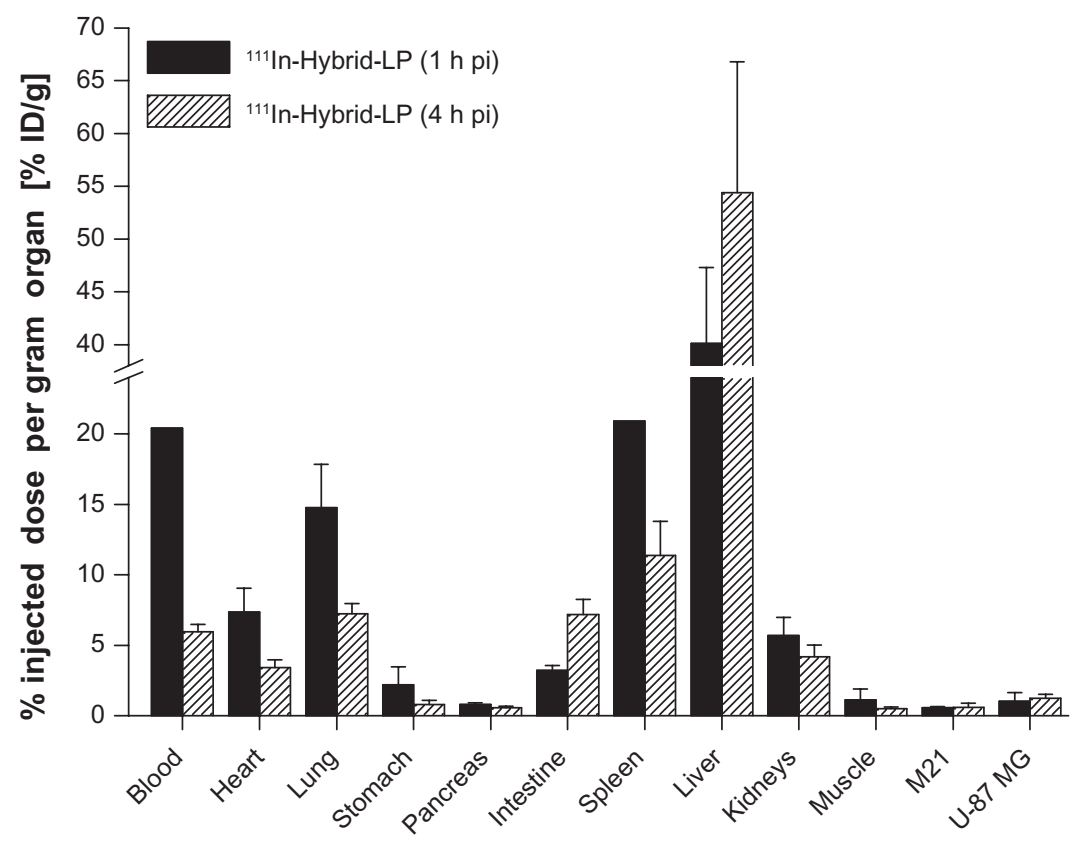

Figure 3 Ex vivo biodistribution of "'In-Hybrid-LP in BALB/c nude mice bearing M2I and U-87 MG tumor xenografts, respectively, at I and 4 hours post-injection ( $\mathrm{n}=\mathrm{I0}$ ). Notes: Values are expressed as the percentage of injected dose per gram (\% ID/g; means \pm standard deviation).

Abbreviations: \% ID/g, percentage injected dose per gram organ; "'In, indium-III; h, hour; LP, liposome; pi, post-injection; RGD, arginine-glycine-aspartic acid; Hybrid-LP, liposomal nanoparticle carrying an RGD and SP building block; SP, substance P. 


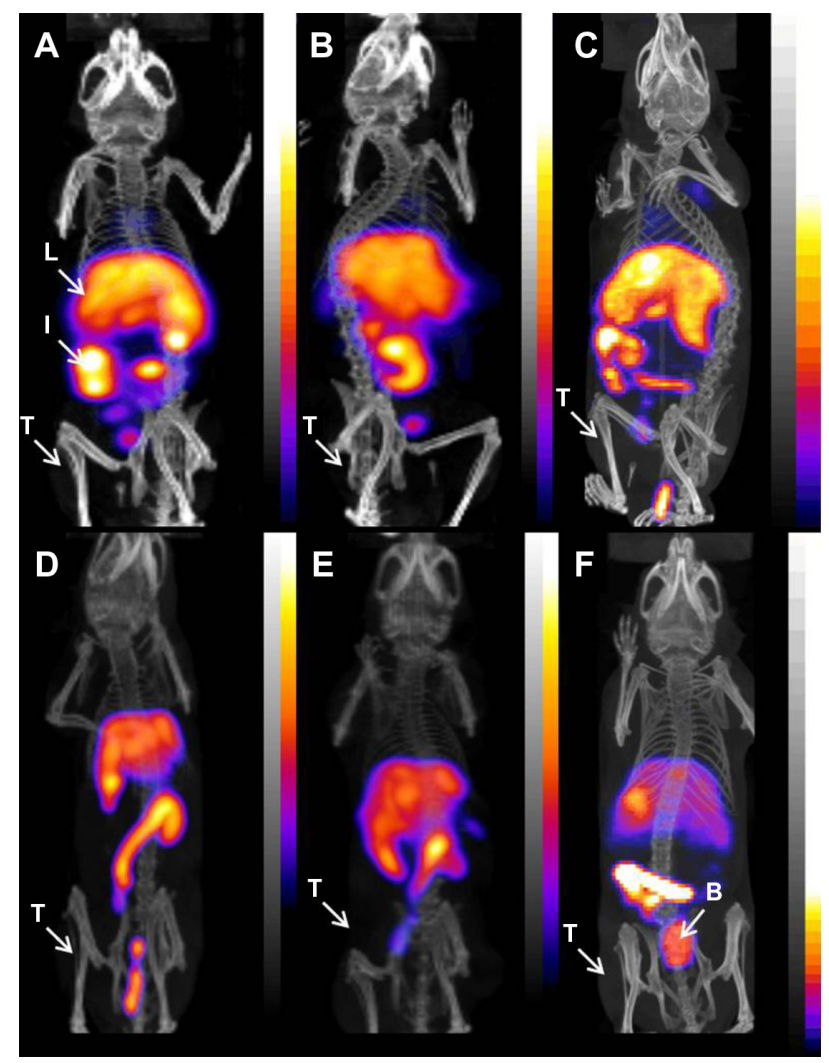

Figure 4 Micro-SPECT/CT images of U-87 MG tumor-xenografted SCID mice after intravenous injection of '"'In-labeled liposomal nanoparticles: (A) RLP, (B) HybridLP, and (C) NoTarget-LP 4 hours after administration; (D) RLP, (E) Hybrid-LP, and (F) NoTarget-LP at 24 hours post-injection (RLP, n=I; Hybrid-LP, n=I; NoTarget$L P, n=I$ ).

Abbreviations: "'In, indium-III; B, bladder; Hybrid-LP, liposomal nanoparticle carrying an RGD and SP building block; I, intestine; L, liver; LP, liposome; NoTargetLP, liposomal nanoparticle with no targeting sequence; RGD, arginine-glycineaspartic acid; RLP, liposomal nanoparticle carrying an RGD building block; SCID, severe combined immunodeficiency; SP, substance P; SPECT/CT, single photon emission computed tomography/computed tomography; T, tumor.

determine and visualize pathologic changes at an early stage, plan the therapy to be given, and further on track the response to therapy. ${ }^{27}$ Most tumors and the surrounding vasculature overexpress numerous markers for functional molecular imaging and targeted therapeutics, thereby providing targets for the delivery of a variety of agents. In this study, based on the promising results of our group with a single-targeted RLP, tumor targeting properties of single-targeted RLP and SP-LP were evaluated in a U-87 MG tumor xenograft mouse model. ${ }^{14}$

The design of multifunctional molecular targeting and imaging probes is an attractive approach because many cancer types simultaneously express multiple receptor types. ${ }^{28}$ Accordingly, we describe here for the first time the in vitro and in vivo evaluation of multifunctional LNPs derivatized with two targeting sequences (RGD and SP). The LNPs were equipped with labels allowing in vitro and in vivo imaging of tumors as well as tumor-induced angiogenesis using multiple imaging modalities (SPECT, fluorescence microscopy, MRI). Dual-targeting with the RGD and SP peptide sequence on the same liposome (Hybrid-LP) has not been described before, and was chosen because dualtargeting constructs have the potential to show synergistic effects in vivo and in vitro compared with their singlemodified versions. ${ }^{29}$ By using these two targeting sequences, we wanted to increase the specificity of the liposomes to reach their pathologic target and accumulate in sufficient amounts while preserving nondiseased tissue. The elevated accumulation at the tumor site and the combination of LNPs with radioactive or imaging labels would lead to improved imaging qualities.

So far, research regarding multitargeted nanoparticles has concentrated on the combination of different antibodies or an antibody and folic acid on the same nanoparticle for drug delivery or diagnostic applications. ${ }^{30-32}$ Multimodal liposomes (radiolabeled and paramagnetic) carrying a RGD-targeting sequence for SPECT/MRI have also been investigated..$^{33}$ Laginha et al reported on two antibodies $(\alpha \mathrm{CD} 19, \alpha \mathrm{CD} 20)$ which were coupled to a liposome carrying the anticancer drug doxorubicin. ${ }^{30}$ These dual-targeted liposomes led to increased uptake in B-cell lymphoma, resulting in highly cytotoxic effects. This positive outcome was also described by Trubetskoy et al who used biotinylated radiolabeled liposomes derivatized with a mixture of modified antibodies. ${ }^{34}$ It was shown in a model system using monolayers with different extracellular antigens and appropriated antibodies that treatment of the target with the mixture of biotinylated antibodies against all target components and also the subsequent binding with the target of biotinylated liposomes via avidin permitted a high liposome concentration on the monolayer. Compared with liposomes derivatized with only one single antibody type, higher binding was achieved. The results of our ex vivo biodistribution study as well as micro-SPECT/CT imaging with dual-targeted LNPs carrying both the RGD and SP peptide sequence did not reveal this synergistic effect in the U-87 MG mouse tumor expressing both $\alpha_{\mathrm{v}} \beta_{3}$ integrins and SP/neurokinin-1 receptors. The ${ }^{111}$ In-Hybrid-LP showed only low accumulation in both the U-87 MG and M21 tumor xenografts (1 hour post-injection). At 4 hours post-injection, uptake in the U-87 MG tumor xenografts was lower than with the single-targeted SP-LP, although this difference was not significant. Interestingly, blood levels of ${ }^{111}$ In-HybridLP were higher, indicating good circulation times of the liposomes. In contrast, for their dual-targeted liposomes 

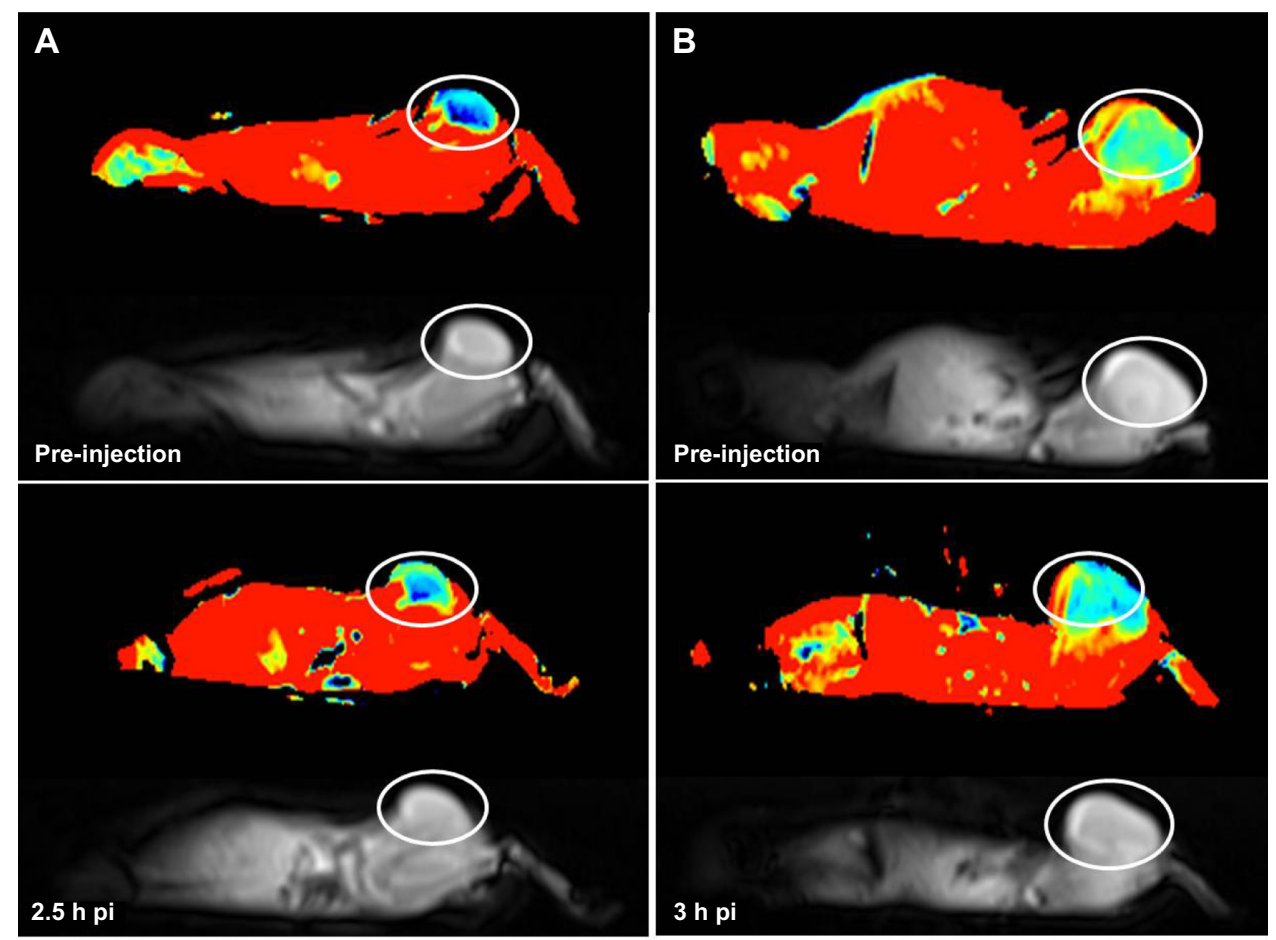

$\mathrm{R} 1(1 / \mathrm{s})$

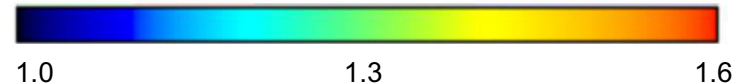

Figure 5 Representative MRI images of tumor-xenografted BALB/c nude mice before and after injection of Gd-RLP or Gd-NoTarget-LP ( $n=2$ ). The increase in RI over time is indicated as a change of color in the ROI. The U-87 MG tumor xenograft is labeled with a white circle. (A) Tumor uptake of Gd-RLP in the U-87 MG tumor xenograft at 2.5 hours post-injection. (B) For Gd-NoTarget-LP no significant uptake in the U-87 MG tumor xenograft could be observed within the 3-hour observation period.

Abbreviations: Gd, gadolinium; h, hour; LP, liposome; MRI, magnetic resonance imaging; NoTarget-LP, liposomal nanoparticle with no targeting sequence; pi, post-injection; RGD, arginine-glycine-aspartic acid; RLP, liposomal nanoparticle carrying an RGD building block; ROI, region of interest.

(galectin-1-specific anginex and an RGD peptide), Kluza et al reported a more rapid blood clearance compared with their single-targeted RGD-liposomes. ${ }^{35}$

The reduced tumor-targeting properties of the ${ }^{111}$ In-Hybrid-LPs could be related to an observation made by Laginha et al who reported a steric hindrance of binding caused by high antibody densities on the liposomes. ${ }^{30}$ Due to the composition of the Hybrid-LP, it cannot be ruled out that the arrangement of RGD and SP building blocks is too close to achieve a synergistic effect in this particular tumor model, even though molar ratios of the two targeting sequences were set at half of the molar ratios in the single-targeted LNPs. Kluza et al did not observe these effects of impaired interaction with the receptors for their dual-targeted anginex/ RGD liposome. ${ }^{36}$ The authors claim that, due to the small size of their targeting sequence, no negative binding interactions between the two sequences are possible. Although the reason for the lack of tumor targeting of the Hybrid-LPs remains unclear and may reflect the limitations of our tumor model, our results stress the importance of obtaining the ideal combination of targeting sequences on the liposomal surface in order to achieve high accumulation of the drug or imaging agent, respectively.

Parallel to the in vivo studies, multifunctional Rhodamine-B-labeled LNPs have been investigated using fluorescence microscopy, where interactions between LNPs and cells can easily be observed. In comparison with the in vivo results, we concluded that steric effects have a minor influence on binding properties since the receptor density is much higher in vitro, offering more targets for binding, and factors like, eg, detection by the mononuclear phagocytic system causing fast blood clearance of the liposomes in vivo, are excluded..$^{30}$ This hypothesis was also supported by the results of the in vitro binding assays for targeted RGDLNPs which showed good binding to isolated $\alpha_{v} \beta_{3}$ integrin receptors. ${ }^{14}$ Basically, all targeted RhB-LNPs showed cell binding to the corresponding tumor cells, whereas for non-targeted RhB-NoTarget-LPs, no cell association was observed and also no fluorescent signal was found on the control cells with any type of the tested RhB-LNPs. Interestingly, RhB-Hybrid-LPs displayed lower binding to cancer cells than the single-targeted versions, indicating 


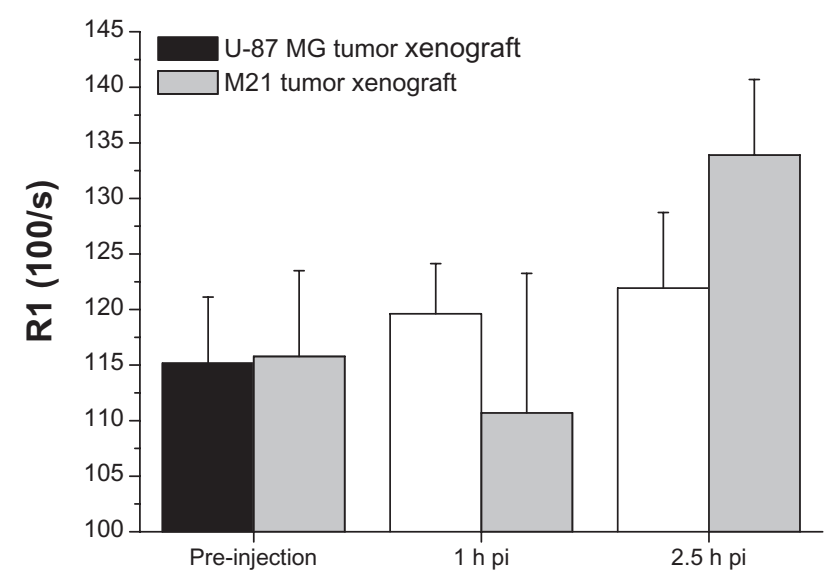

Figure 6 Off-line analysis of the MRI images showing a significant uptake (increase of RI over time) of Gd-RLP in the U-87 MG tumor xenograft within the observation period. For the $\mathrm{M} 2 \mathrm{I}$ tumor xenograft an increase of RI was observed only for the 2.5-hour image.

Note: Values are expressed as means \pm standard deviation.

Abbreviations: Gd, gadolinium; h, hour; MRI, magnetic resonance imaging; pi, postinjection; RGD, arginine-glycine-aspartic acid; RLP, liposomal nanoparticle carrying an RGD building block.

no synergistic or additive effect, but confirming the in vivo results of the biodistribution and micro-SPECT/CT imaging studies with the ${ }^{111}$ In-labeled LNPs.

In the preliminary MRI study, a single-targeted RGDliposome (Gd-RLP) and a nontargeted Gd-No-Target-LP with a high payload of the paramagnetic metal gadolinium were investigated for noninvasive tumor angiogenesis imaging. This approach has been described by Sipkins et al with paramagnetic vesicles targeting $\alpha_{v} \beta_{3}$ integrin expression via a monoclonal antibody. ${ }^{37}$ We wanted to take advantage of the better spatial resolution and more precise anatomic localization of MRI in comparison with positron emission tomography or SPECT. ${ }^{38}$ The results of the MRI experiments with BALB/c nude mice bearing both the U-87 MG and M21 tumor xenografts were promising, in that they revealed that the Gd-NoTarget-LP was mainly taken up by the liver and did not accumulate within the tumor tissue. In contrast with that, the single-targeted Gd-RLP was taken up in both tumor xenografts, and liver uptake increased slowly within the 3-hour observation time, suggesting a long circulation time of the liposome. In a similar study, Flament et al used liposome-encapsulated paramagnetic contrast agents conjugated with an RGD peptide for targeting of $\alpha_{v} \beta_{3}$ integrin receptors in U-87 MG mouse brain tumors using MRI. ${ }^{39}$ The investigators demonstrated high-sensitivity in vivo MRI of angiogenesis in mouse brain tumors which additionally supports the obtained results showing selective uptake of the targeted RLP in both tumor xenografts studied. It should be pointed out that the gadolinium loading was not optimized for this preliminary study; however, interesting in this context is the fact that localization of the tumor with MRI was better as compared with radiolabeled RLP using micro-SPECT/CT imaging. The reason for this could be related to the different amounts of LNPs used in both studies, and further studies are warranted to investigate this effect.

\section{Conclusion}

The evaluation of multifunctional LNPs for noninvasive imaging of tumors and $\alpha_{\mathrm{v}} \beta_{3}$ integrin receptor expression on angiogenic tumor vessels using SPECT, fluorescence microscopy, and MRI is described in this study. A dual-targeting strategy was used to design liposomes containing both an RGD peptide and the neuropeptide SP as targeting ligands as well as either the radioactive label ${ }^{111} \mathrm{In}$, Rhodamine-B, or gadolinium for multiple imaging applications. Our findings are a proof of principle of the potential of LNPs as multifunctional targeting agents and indicate that the tested liposomes have potential for further optimization. Even though no synergistic effects could be obtained for hybrid LNPs carrying both the RGD and SP peptide sequence, improved multifunctional LNPs with other combinations of targeting sequences may be used for more efficient multireceptor targeting and imaging of malignant tissue in vivo. Multifunctionality could lead to more personalized medicine by using LNPs equipped with a variety of drugs, imaging labels, and targeting structures.

\section{Acknowledgments}

The Austrian Nano-Initiative cofinanced this work as part of the Nano-Health project (number 0200), with the subprojects, NANO-NUC and NANO-LIPO, being financed by the Austrian Research Promotion Agency FFG (project NanoHealth, number 819721). Peter A Knetsch, Department of Nuclear Medicine (Innsbruck Medical University, Austria), is acknowledged for his assistance with the biodistribution studies. We thank Julia Schindelar, Max Perutz Laboratories (University of Vienna, Austria), for performing the fluorescence imaging studies. Ciara Finucane, Jerome Burnet, Julius Leyton, and Julie Foster assisted in the micro-SPECT/ CT imaging studies carried out at the Centre for Molecular Oncology, Barts Cancer Institute, Queen Mary University of London (London, UK). We would also like to express our thanks to Gudrun C Thurner, Department of Radiology (Innsbruck Medical University, Austria), and Glen Perera, Institute of Pharmacy (University of Innsbruck, Austria), for their assistance with the photon correlation spectroscopy and zeta potential measurements. Dr David A Cheresh and the 
Scripps Research Institute (La Jolla, CA, USA) are acknowledged for providing the human melanoma cells.

\section{Disclosure}

The authors report no conflict of interest, and are alone responsible for the content and writing of this paper.

\section{References}

1. Li S, Goins B, Zhang L, Bao A. Novel multifunctional theranostic liposome drug delivery system: construction, characterization, and multimodality MR, near-infrared fluorescent, and nuclear imaging. Bioconjug Chem. 2012;23:1322-1332.

2. Kalia M. Personalized oncology: recent advances and future challenges. Metabolism. 2013;62 Suppl 1:11-14.

3. Kircher MF, Hricak H, Larson SM. Molecular imaging for personalized cancer care. Mol Oncol. 2012;6:182-195.

4. Wender PA, Cooley CB, Geihe EI. Beyond cell penetrating peptides: designed molecular transporters. Drug Discov Today Technol. 2012;9: e49-e55.

5. Torchilin VP. Recent advances with liposomes as pharmaceutical carriers. Nat Rev Drug Discov. 2005;4:145-160.

6. Abuchowski A, Van Es T, Palczuk NC, Davis FF. Alteration of immunological properties of bovine serum albumin by covalent attachment of polyethylene glycol. J Biol Chem. 1977;252:3578-3581.

7. Allen C, Dos Santos N, Gallagher R, et al. Controlling the physical behavior and biological performance of liposome formulations through use of surface grafted poly(ethylene glycol). Biosci Rep. 2002;22: $225-250$.

8. Torchilin VP. Tumor delivery of macromolecular drugs based on the EPR effect. Adv Drug Deliv Rev. 2011;63:131-135.

9. Lattin JR, Belnap DM, Pitt WG. Formation of eLiposomes as a drug delivery vehicle. Colloids Surf B Biointerfaces. 2012;89:93-100.

10. Shokeen M, Pressly ED, Hagooly A, et al. Evaluation of multivalent, functional polymeric nanoparticles for imaging applications. ACS Nano. 2011;5:738-747.

11. Pasqualini R, Arap W, McDonald DM. Probing the structural and molecular diversity of tumor vasculature. Trends Mol Med. 2002;8: 1335-1347.

12. Strijkers GJ, Kluza E, Van Tilborg GA, et al. Paramagnetic and fluorescent liposomes for target-specific imaging and therapy of tumor angiogenesis. Angiogenesis. 2010;13:161-173.

13. Ruoslahti E, Pierschbacher MD. Arg-Gly-Asp: a versatile cell recognition signal. Cell. 1986;44:517-518.

14. Rangger C, Helbok A, Von Guggenberg E, et al. Influence of PEGylation and RGD loading on the targeting properties of radiolabeled liposomal nanoparticles. Int J Nanomedicine. 2012;7:5889-5900.

15. Chen X, Park R, Hou Y, et al. MicroPET imaging of brain tumor angiogenesis with ${ }^{18} \mathrm{~F}$-labeled PEGylated RGD peptide. Eur J Nucl Med Mol Imaging. 2004;31:1081-1089.

16. Muñoz M, Rosso M, Coveñas R. The NK-1 receptor: a new target in cancer therapy. Curr Drug Targets. 2011;12:909-921.

17. Palma C. Tachykinins and their receptors in human malignancies. Curr Drug Targets. 2006;7:1043-1052.

18. Hennig IM, Laissue JA, Horisberger U, Reubi JC. Substance-P receptors in human primary neoplasms: tumoral and vascular localization. Int $J$ Cancer. 1995;61:786-792.

19. Rosso M, Muñoz M, Berger M. The role of neurokinin-1 receptor in the microenvironment of inflammation and cancer. ScientificWorldJournal. 2012;2012:381434.

20. Ogo H, Kuroyanagi N, Inoue A, et al. Human astrocytoma cells (U-87 MG) exhibit a specific substance $\mathrm{P}$ binding site with the characteristics of an NK-1 receptor. J Neurochem. 1996;67:1813-1820.
21. Liu Z, Jia B, Zhao H, Chen X, Wang F. Specific targeting of human integrin $\alpha_{\mathrm{v}} \beta_{3}$ with ${ }^{111}$ In-labeled Abegrin ${ }^{\mathrm{TM}}$ in nude mouse models. Mol Imaging Biol. 2011;13:112-120.

22. Cai W, Chen X. Nanoplatforms for targeted molecular imaging in living subjects. Small. 2007;3:1840-1854.

23. Bangham AD, Standish MM, Watkins JC. Diffusion of univalent ions across the lamellae of swollen phospholipids. J Mol Biol. 1965;13: 238-252.

24. Helbok A, Decristoforo C, Dobrozemsky G, et al. Radiolabeling of lipid-based nanoparticles for diagnostics and therapeutic applications: a comparison using different radiometals. J Liposome Res. 2010;20: 219-227.

25. Rofsky NM, Lee VS, Laub G, et al. Abdominal MR imaging with a volumetric interpolated breath-hold examination. Radiology. 1999;212:876-884.

26. Cheng HL, Wright GA. Rapid high-resolution T(1) mapping by variable flip angles: accurate and precise measurements in the presence of radiofrequency field inhomogeneity. Magn Reson Med. 2006;55: 566-574.

27. Weissleder R, Mahmood U. Molecular imaging. Radiology. 2001;219: 316-333.

28. Liu Z, Wang F. Dual-targeted molecular probes for cancer imaging. Curr Pharm Biotechnol. 2010;11:610-619.

29. Takara K, Hatakeyama H, Ohga N, Hida K, Harashima H. Design of a dual-ligand system using a specific ligand and cell penetrating peptide, resulting in a synergistic effect on selectivity and cellular uptake. Int J Pharm. 2010;396:143-148.

30. Laginha K, Mumbengegwi D, Allen T. Liposomes targeted via two different antibodies: assay, B-cell binding and cytotoxicity. Biochim Biophys Acta, 2005;1711:25-32.

31. Saul JM, Annapragada AV, Bellamkonda RV. A dual-ligand approach for enhancing targeting selectivity of therapeutic nanocarriers. J Control Release. 2006;114:277-287.

32. McAteer MA, Schneider JE, Ali ZA, et al. Magnetic resonance imaging of endothelial adhesion molecules in mouse atherosclerosis using dualtargeted microparticles of iron oxide. Arterioscler Thromb Vasc Biol. 2008;28:77-83.

33. De Vries A, Kok MB, Sanders HM, Nicolay K, Strijkers GJ, Gruell H. Multimodal liposomes for SPECT/MR imaging as a tool for in situ relaxivity measurements. Contrast Media Mol Imaging. 2012;7: $68-75$.

34. Trubetskoy VS, Berdichevsky VR, Efremov EE, Torchilin VP. On the possibility of the unification of drug targeting systems. Studies with liposome transport to the mixtures of target antigens. Biochem Pharmacol. 1987;36:839-842.

35. Kluza E, Jacobs I, Hectors SJ, et al. Dual-targeting of $\alpha_{v} \beta_{3}$ and galectin-1 improves the specificity of paramagnetic/fluorescent liposomes to tumor endothelium in vivo. J Control Release. 2012;158:207-214.

36. Kluza E, Van Der Schaft D, Hautvast P, et al. Synergistic targeting of $\alpha_{v} \beta_{3}$ integrin and galectin-1 with heteromultivalent paramagnetic liposomes for combined MR imaging and treatment of angiogenesis. Nano Lett. 2010;10:52-58.

37. Sipkins DA, Cheresh DA, Kazemi MR, Nevin LM, Bednarski MD, Li K. Detection of tumor angiogenesis in vivo by alphaVbeta3-targeted magnetic resonance imaging. Nat Med. 1998;4:623-626.

38. Li W, Su B, Meng S, et al. RGD-targeted paramagnetic liposomes for early detection of tumor: in vitro and in vivo studies. Eur J Radiol. 2011;80:598-606.

39. Flament J, Geffroy F, Medina C, et al. In vivo CEST MR imaging of U87 mice brain tumor angiogenesis using targeted LipoCEST contrast agent at 7 T. Magn Reson Med. 2013;69:179-187. 
International Journal of Nanomedicine

Dovepress

\section{Publish your work in this journal}

The International Journal of Nanomedicine is an international, peerreviewed journal focusing on the application of nanotechnology in diagnostics, therapeutics, and drug delivery systems throughou the biomedical field. This journal is indexed on PubMed Central, MedLine, CAS, SciSearch ${ }^{\circledR}$, Current Contents ${ }^{\circledR} /$ Clinical Medicine,
Journal Citation Reports/Science Edition, EMBase, Scopus and the Elsevier Bibliographic databases. The manuscript management system is completely online and includes a very quick and fair peer-review system, which is all easy to use. Visit http://www.dovepress.com/ testimonials.php to read real quotes from published authors.

Submit your manuscript here: http://www.dovepress.com/international-journal-of-nanomedicine-journal 